\title{
Understanding the enhanced stability of bromide substitution in lead iodide perovskites
}

Alex Aziz, ${ }^{\dagger}$ Nicholas Aristidou, ${ }^{\ddagger}$ Xiangnan $B u,{ }^{\ddagger}$ Robert J. E. Westbrook, ${ }^{\ddagger}$ Saif A. Haque $^{{ }^{*}}$ and M. Saiful Islam ${ }^{+*}$

${ }^{\dagger}$ Department of Chemistry, University of Bath, Bath, BA2 7AY, UK.

‡Department of Chemistry, Imperial College London, London SW7 2AZ, UK.

E-mail: m.s.islam@bath.ac.uk ${ }^{1^{*}}$; s.a.haque@imperial.ac.uk ${ }^{2^{*}}$ 


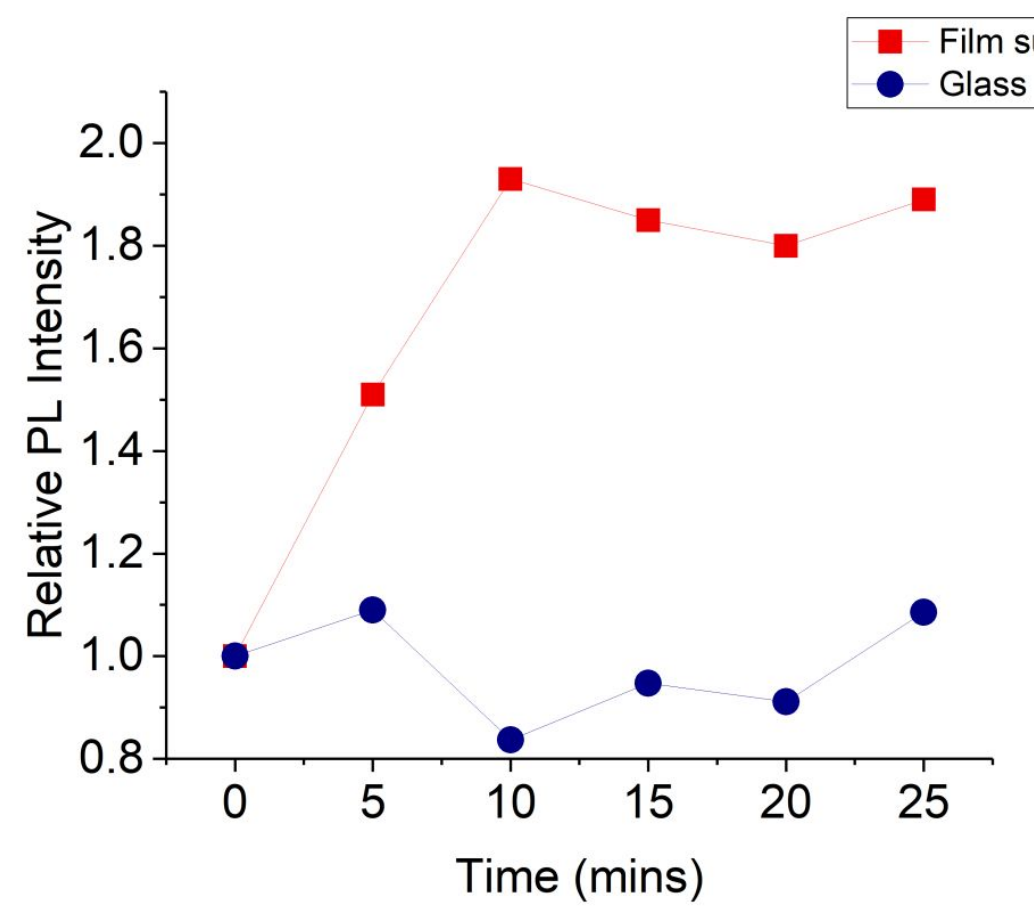

Figure S1. Relative PL Intensity of $\mathrm{MAPbBr}_{3}$ when excited from the film surface (red) and the substrate (navy). Films were measured fresh and at subsequent time intervals after exposure to oxygen.

Table S1. Normalised weight increase for the selected film compositions, of MAPb( $\mathrm{I}_{1}$ $\left.{ }_{x} \mathrm{Br}_{x}\right)_{3}$ as a function of exposure to dry air flux against time.

\begin{tabular}{ccc}
$\boldsymbol{x}$ & $\begin{array}{c}\text { Normalised weight } \\
\mathbf{t}=\mathbf{1 8 0 s}\end{array}$ & \% Increase \\
\hline 0.0 & 1.0165 & 1.65 \\
0.2 & 1.0147 & 1.47 \\
0.5 & 1.0106 & 1.06 \\
0.75 & 1.0083 & 0.83 \\
1.0 & 1.0002 & 0.02 \\
\hline
\end{tabular}

Table S2. Degradation reaction energies, based on reaction (1) and (2).

\begin{tabular}{ccc}
\hline \multirow{2}{*}{ in $\mathbf{M A P b}\left(\mathbf{I}_{\mathbf{1}-\boldsymbol{x}} \mathbf{B r}_{\boldsymbol{x}}\right)_{\mathbf{3}}$} & \multicolumn{2}{c}{ Reaction pathway } \\
\cline { 2 - 3 } & $\mathbf{1}$ & $\mathbf{2}$ \\
\hline 0 & 1.56 & -1.39 \\
0.25 & 1.70 & -1.23 \\
0.50 & 1.90 & -1.06 \\
1 & 2.78 & -0.18 \\
& 4.98 & 2.00 \\
\hline
\end{tabular}




\section{Theoretical representation of the disordered solids.}

Disorder for the solid solutions $\mathrm{MAPb}\left(\mathrm{I}_{1-x} \mathrm{Br}_{x}\right)_{3}$ was represented by a symmetryadapted ensemble of configurations using the methodology implemented in the SOD (Site Occupancy Disorder program. ${ }^{1}$ A list of symmetrically inequivalent configurations is generated for which energies are evaluated using periodic boundary conditions. The occurrence of a particular configuration is described by a Boltzmann-like probability, which is calculated from its energy $\left(E_{m}\right)$ and its degeneracy $\left(\Omega_{m}\right)$ :

$$
P_{m}=\frac{\Omega_{m}}{Z} \exp \left(-\frac{E_{m}}{k_{\mathrm{B}} T}\right)
$$

where index $m$ take the values $1, \ldots, M$ (M is the total number of inequivalent configurations), $k_{\mathrm{B}}$ is the Boltzmann's constant, and $\mathrm{Z}$ is the partition function, ensuring that the sum of all the probabilities equals 1 . SOD calculates only the energies of configurations that are symmetrically inequivalent. To reduce the number of symmetry inequivalent configurations, the methylammonium cation is taken as a being spherical and fully symmetrical, and a full structural optimization is performed for each of the generated inequivalent configurations following the method reported by Brivio et al. ${ }^{2}$

Table S3: The total number of possible configurations and the number of inequivalent configurations from each cell composition in the tetragonal unit cell.

\begin{tabular}{ccc}
\hline $\boldsymbol{x}$ in $4\left[\mathbf{M A P b}\left(\mathbf{I}_{1-x} \mathrm{Br}_{\boldsymbol{x}}\right)_{3}\right]$ & Total number of configurations & $\begin{array}{c}\text { Number of inequivalent } \\
\text { configurations }(\boldsymbol{M})\end{array}$ \\
\hline 0 & 1 & 1 \\
1 & 2 & 2 \\
3 & 220 & 16 \\
6 & 924 & 75 \\
9 & 220 & 16 \\
12 & 1 & 1 \\
\hline
\end{tabular}


We are now able to calculate the average lattice parameters as a function of composition, which are calculated in the tetragonal configuration (Figure S2). The free energies and enthalpies of mixing are also calculated as a weighted average over all the configurations (Figure S3). ${ }^{1}$ The positive $\Delta \mathrm{H}$ indicates that the fully ordered system $(0 \mathrm{~K})$ is not stable versus the end member phases indicating a miscibility gap, although the mixed phases can be stabilized by entropic affects. This is a result of the small differences in energies between the configurations. The lowest 10 configurations in the 50:50 phase differ in energy by just $34 \mathrm{meV}$ with a difference in energy of $160 \mathrm{meV}$ across all 75 inequivalent configurations. This leads to a negative free energy of mixing at $300 \mathrm{~K}$ (Figure S3). Although it is noted that phase separation due to light-induced spinodal decomposition has also been reported in previous calculations. ${ }^{2}$

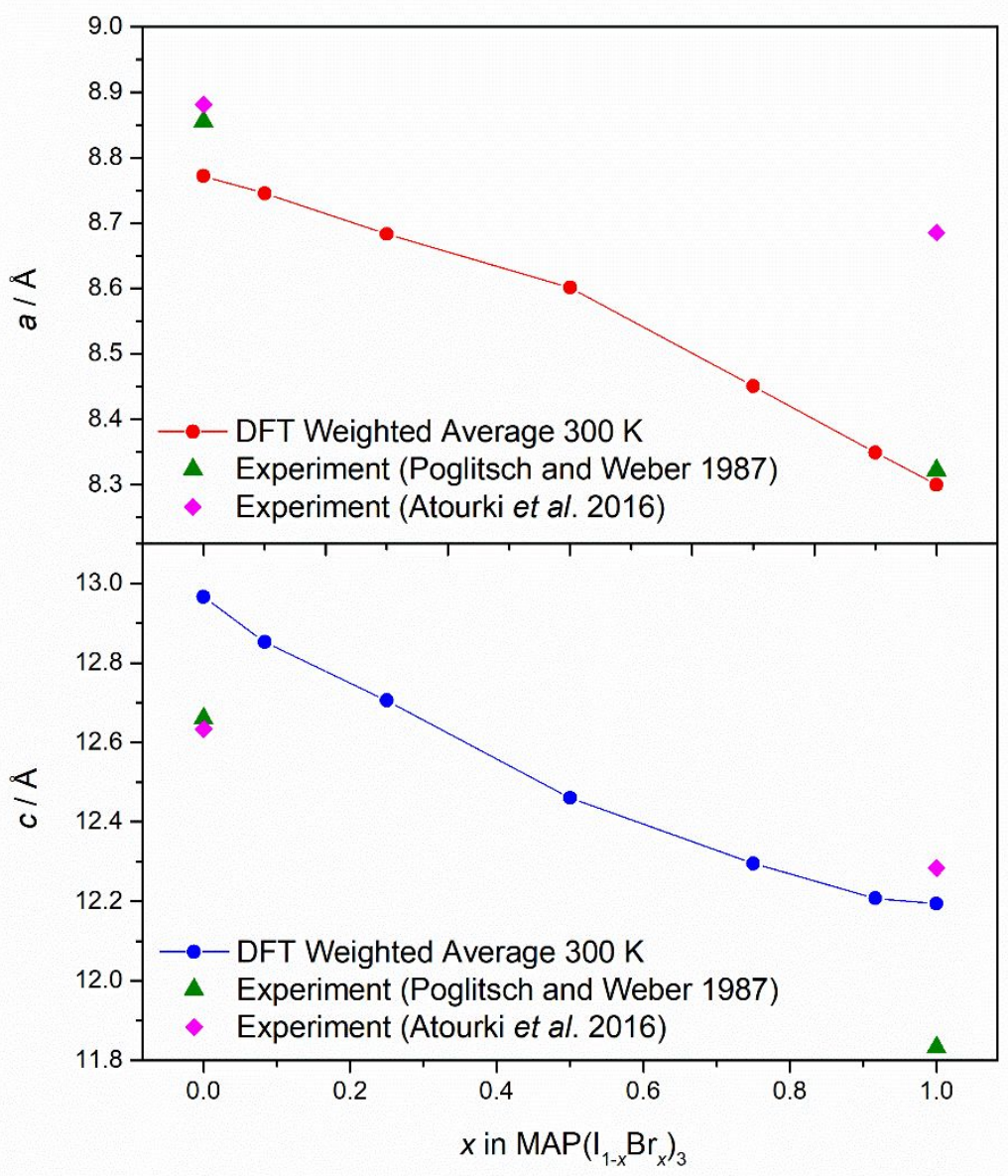


Figure S2. Average lattice parameters as a function of composition. Disorder is represented by a symmetry-adapted ensemble of configurations using the methodology implemented in the SOD (Site Occupancy Disorder) program ${ }^{1}$ and compared to experiment. ${ }^{3,4}$

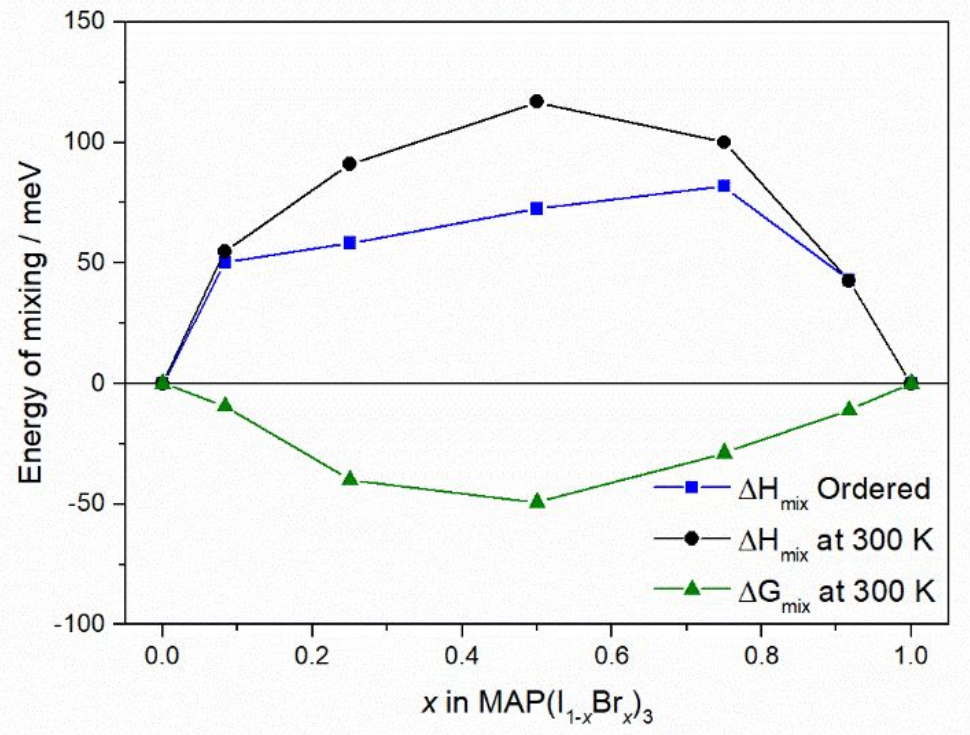

Figure S3: Calculated enthalpy and free energy of mixing as a function of the Br content 
a)
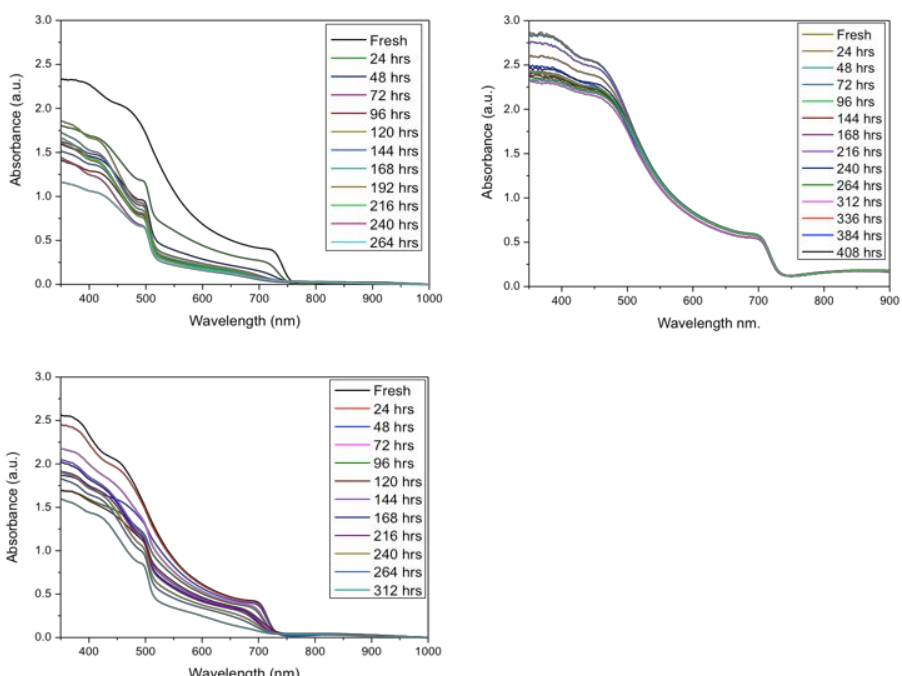

b)
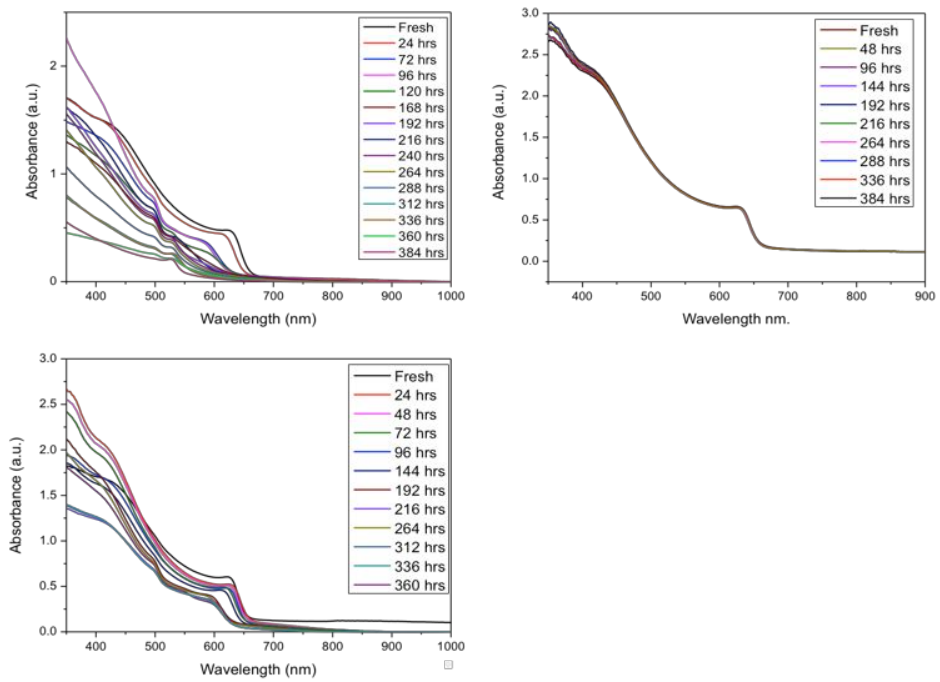

c)
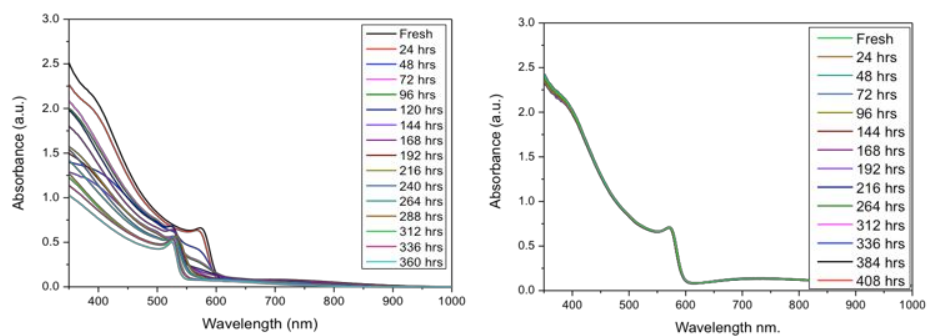

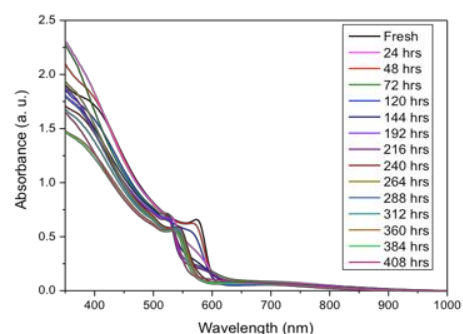

Figure S4. Raw absorbance data collected for perovskite films (a) $20 \%$ doped $\mathrm{Br}$, (b) $50 \% \mathrm{Br}$ and (c) 75\%. No coating (top left), $0.01 \mathrm{M}$ (top right) and 0.001M (bottom left) MAI salt treatment. Films were measured fresh and then at subsequent time intervals after exposure to dry air flux and ambient illumination. 
Table S4. Calculated hydrogen --X distances in $\mathrm{MAPbI}_{3}$ and $\mathrm{MAPbBr}_{3}$. The $\mathrm{X}-\mathrm{CH}_{3}$ distances of over $3 \AA$ show they do not form H-bonds in these phases.

\begin{tabular}{c|ccc|ccc}
\hline Composition & \multicolumn{3}{|c|}{$\mathbf{X}-\mathbf{N H}_{3}^{+}(\AA)$} & \multicolumn{3}{c}{$\mathbf{X}-\mathbf{C H}_{\mathbf{3}}(\AA)$} \\
\hline $\mathrm{MAPbBr}_{3}$ (tetragonal) & 2.39 & 2.39 & 2.73 & 3.01 & 3.04 & 2.93 \\
$\mathrm{MAPbBr}_{3}$ (cubic) & 2.45 & 2.45 & 2.67 & 3.09 & 3.09 & 3.34 \\
$\mathrm{MAPbI}_{3}$ (tetragonal) & 2.61 & 2.59 & 2.99 & 3.09 & 3.09 & 3.28 \\
\hline
\end{tabular}

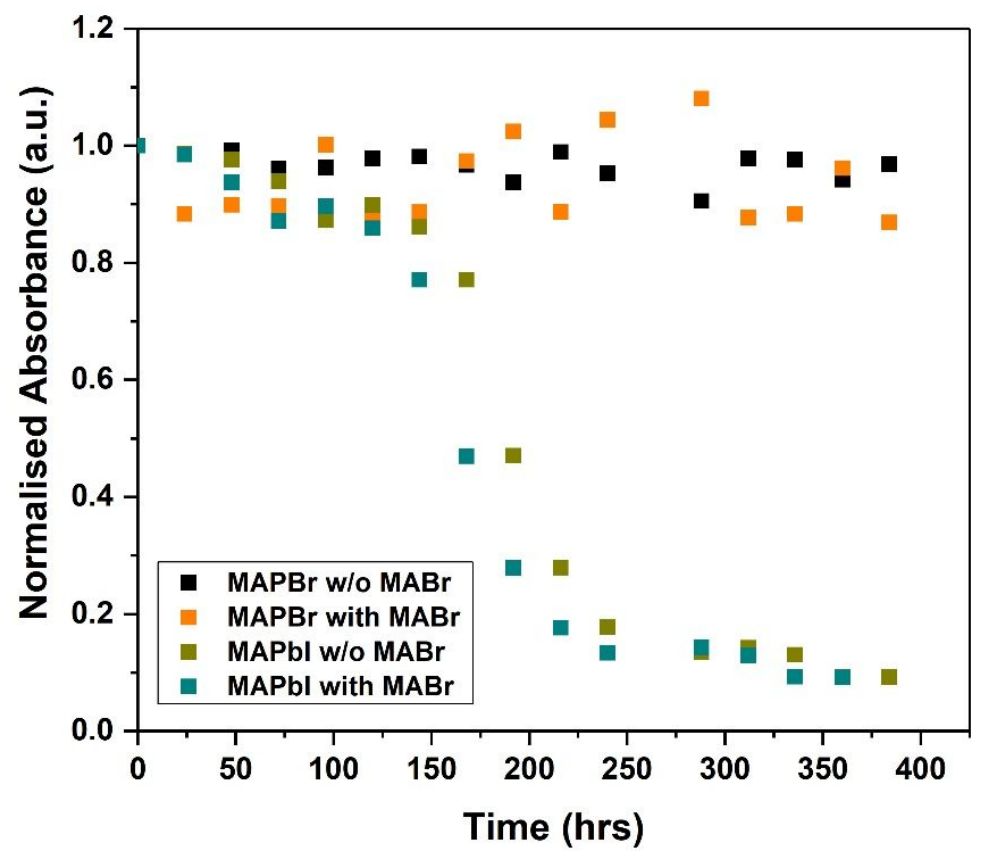

Figure S5. UV-vis absorption spectroscopy was used to probe the stability of $\mathrm{MAPbBr}_{3}$ and $\mathrm{MAPbI}_{3}$ films with and without $\mathrm{MABr}$ salt coatings.

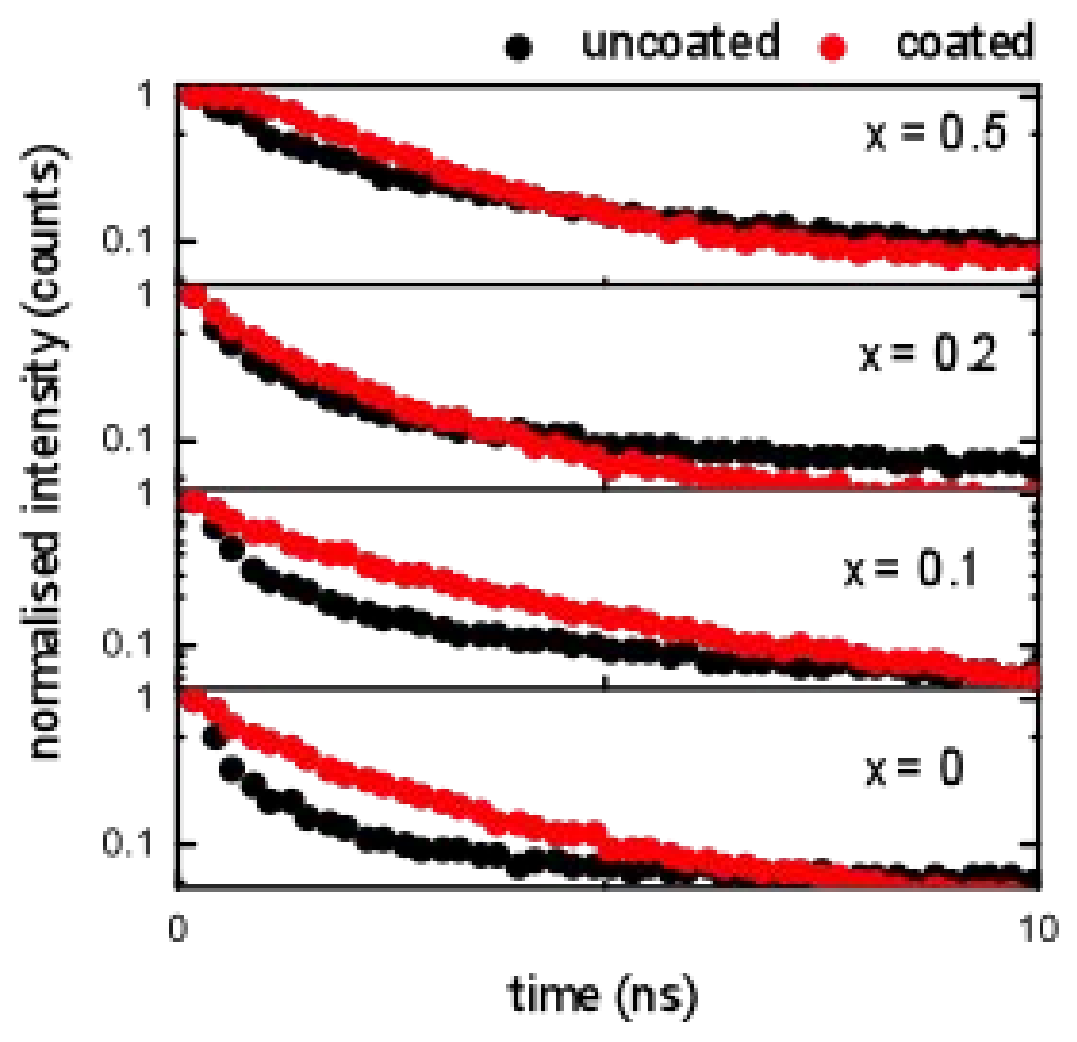


Figure S6. Time-resolved photoluminescence spectra for film compositions of $x=0, x=0.1, x=0.2$ and $x=0.5$ with and without a salt coating of $0.01 M$ MAI. Salt coating concentration increases lifetime emission intensity to a greater degree as the content of bromide decreases. 

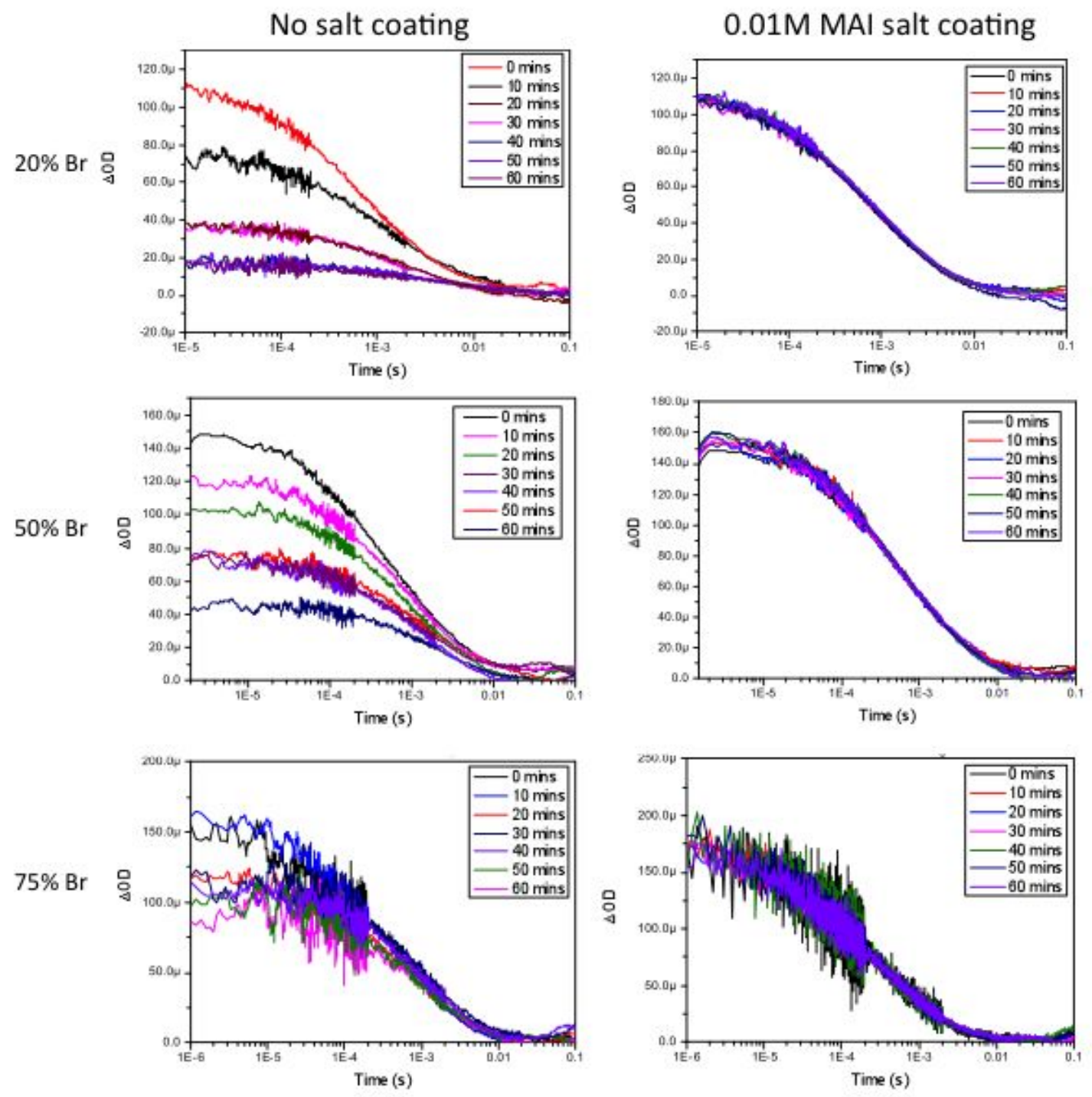

Figure S7. Raw transient absorption spectroscopy data for $\operatorname{MAPb}\left(\mathrm{I}_{1-x} B r_{x}\right)_{3}$, showing the obtained kinetic decay traces for films with $x=0.2,0.5$ and 0.75 with and without a $0.01 M$ MAI salt coating. Probed at $1600 \mathrm{~nm}$ (tracking concentration of Spiro-OMeTAD hole polarons) following excitation at $458 \mathrm{~nm}$. 


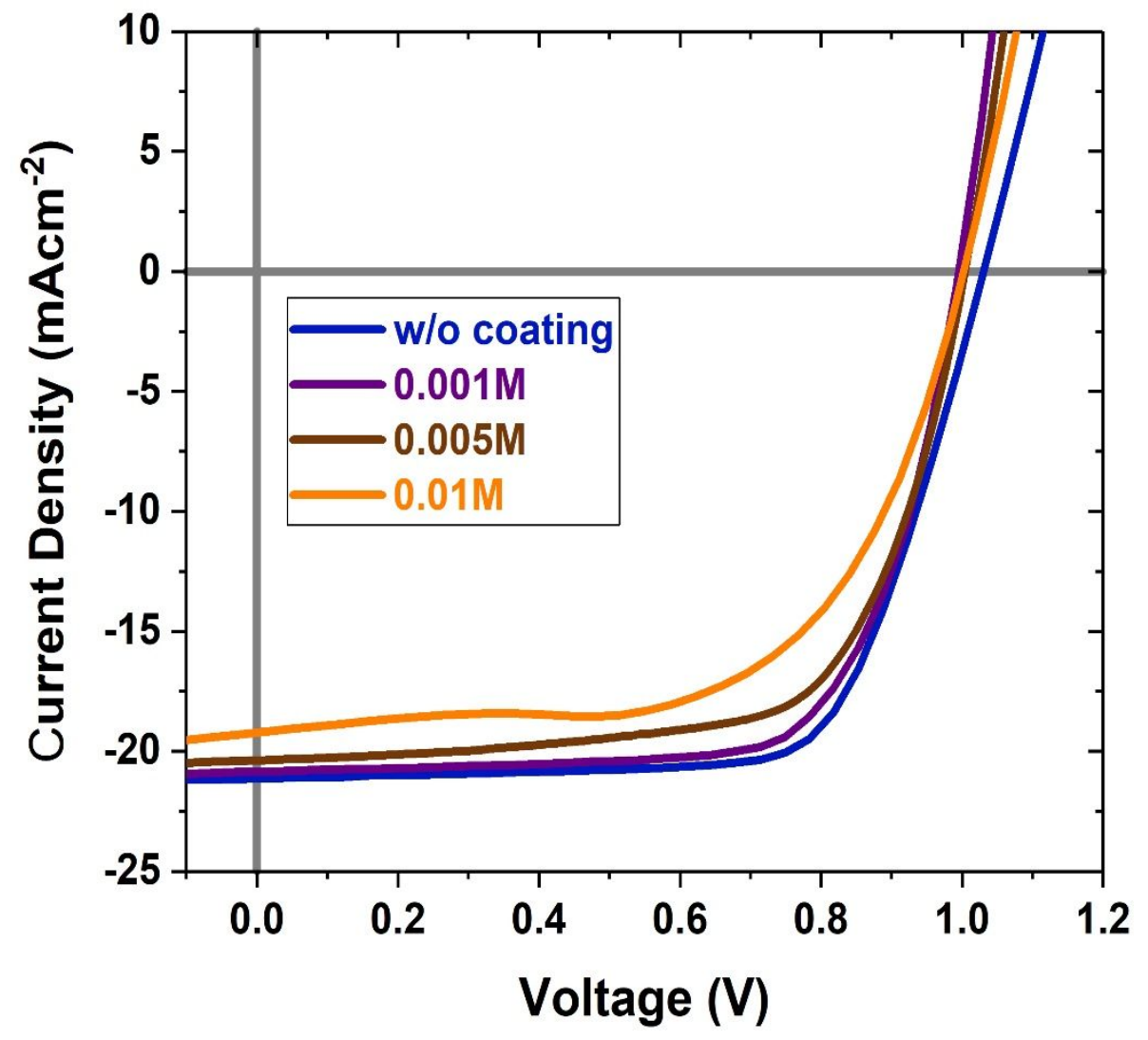

Figure S8. Current-voltage curves of $\operatorname{MAPb}\left(\mathrm{I}_{1-x} B r_{x}\right)_{3}(x=0.2)$ perovskite devices with different concentrations of MAI coating.

Table S5. Photovoltaic parameters for devices based on $\operatorname{MAPb}\left(\mathrm{I}_{1-x} B r_{x}\right)_{3}$ perovskite films $(x=0.2)$ without and with different MAI salt concentrations.

\begin{tabular}{c|ccccc}
\hline $\begin{array}{c}\text { MAI } \\
\begin{array}{c}\text { Concentration } \\
\text { / M }\end{array}\end{array}$ & Pixel Area $\left(\mathbf{c m}^{2}\right)$ & $\mathbf{V}_{\text {oc }}(\mathbf{V})$ & $\left.\mathbf{J}_{\text {sc }}(\mathbf{m A c m})^{-2}\right)$ & FF & PCE (\%) \\
\hline 0 & 0.12 & 1.03 & 21.14 & 0.70 & 15.26 \\
0.001 & 0.12 & 1.00 & 20.85 & 0.70 & 14.53 \\
0.005 & 0.12 & 1.00 & 20.38 & 0.67 & 13.70 \\
0.01 & 0.12 & 1.00 & 19.22 & 0.61 & 11.64 \\
\hline
\end{tabular}




\section{References}

(1) Grau-Crespo, R.; Hamad, S.; Catlow, C. R. A.; de Leeuw, N. H., Symmetry-adapted configurational modelling of fractional site occupancy in solids. J. Phys. Condens. Matter 2007, $19(25), 256201$.

(2) Brivio, F.; Caetano, C.; Walsh, A., Thermodynamic Origin of Photoinstability in the $\mathrm{CH}_{3} \mathrm{NH}_{3} \mathrm{~Pb}\left(\mathrm{I}_{1-x} \mathrm{Br}_{x}\right)_{3}$ Hybrid Halide Perovskite Alloy. J. Phys. Chem. Lett. 2016, 7 (6), $1083-$ 1087.

(3) Poglitsch, A.; Weber, D., Dynamic disorder in methylammoniumtrihalogenplumbates(II) observed by millimeter-wave spectroscopy. J. Chem. Phys. 1987, 87 (11), 6373-6378.

(4) Atourki, L.; Vega, E.; Mari, B.; Mollar, M.; Ahsaine, H. A.; Bouabid, K.; Ihlal, A., Role of the chemical substitution on the structural and luminescence properties of the mixed halide perovskite thin $\operatorname{MAPbI}_{(3-x)} \operatorname{Br}_{(x)}(0<=\mathrm{x}<=1)$ films. Appl. Surf. Sci. 2016, 371, 112-117. 